\title{
Dynamic Clustering
}

\author{
Abdelhamid Bouchachia
}

Received: 1 August 2012/Accepted: 2 August 2012/Published online: 11 August 2012

(C) Springer-Verlag 2012

Clustering is a key technique in pattern recognition, data mining, and knowledge discovery. The aim is to uncover the (hidden) structure underlying a given collection of objects. Due to its importance, there is a continuous research work on new methodologies to improve the available ones. Moreover, there is a clear awareness among researchers and practitioners of the necessity to investigate novel approaches that are more appropriate for many new clustering problems arising in diverse situations and requiring special treatment.

This special issue aims at shedding light on dynamic clustering. This particular type of clustering embraces different scenarios: dynamic features, dynamic data objects and dynamic clusters. Many challenges can be envisioned with each of these clustering scenarios especially in presence of non-stationarity, data drift and shift that take various change rates (slow, medium, fast, random, gradual, abrupt and cyclic).

Dynamic clustering is also relevant to many other clustering situations involving very large data, data streams, incomplete data, noisy data, unbalanced data, and structured data. This makes the challenges quite interesting. Moreover, because clustering can also be just one step in a multi-step complex system, its importance grows. In applications like control, artificial vision, surveillance, etc., clustering quality is vital for the well-functioning of the whole multi-step system. Adding the time dimension to such applications renders clustering dynamic and hence challenging. For instance, in ubiquitous environments, dynamic clustering of distributed, parallel, heterogeneous

A. Bouchachia $(\square)$

Smart Technology Research Centre,

Bournemouth University, Dorset, UK

e-mail: abouchachia@bournemouth.ac.uk and spatio-temporal data is a particularly interesting problem for a number of applications.

Due to the versatility of dynamic clustering, the present special issue intends to provide a wide look on the latest advances in dynamic clustering putting focus on online (one-pass), real-time and incremental scenarios that involve settings of dynamic features, dynamic objects and dynamic clusters. It also targets related issues and relevant applications to dynamic clustering.

Dynamic clustering as a form of unsupervised online/ incremental machine learning considers two concepts: (1) incrementality of the learning methods to devise the clustering model and (2) self-adaptation of the learned model (parameters and structure). Hence, incrementality tackles the problem of time-intensive re-training and memory constraints. As a consequence, dynamic aspects (e.g., behavior, structural elements) of the model to be learned can be captured via adaptation of the current model.

Dynamic (online/incremental) clustering has been attracting the attention of the many research communities (like data mining, pattern recognition, fuzzy theory, control, machine learning, etc.) over the recent years, hence the motivation behind organizing the present special issue. This latter consists of the following papers:

Paper 1 by Fan and Bouguila titled "Online Variational Learning of Finite Dirichlet Mixture Models" introduces an online variational inference algorithm for finite Dirichlet mixture models learning. Relying on an online variational Bayes framework, the structure and the parameters of the Dirichlet mixture model are continuously updated. The proposed algorithm is evaluated on a range of data sets.

Paper 2 by Lughofer titled "A Dynamic Split-andMerge Approach for Evolving Cluster Models" presents a dynamic clustering algorithm which is equipped with dynamic split-and-merge operations and which is dedicated 
to incremental clustering of data streams. The proposed dynamic clustering algorithm is evaluated on high-dimensional streaming data sets to show its efficiency.

Paper 3 titled "The Use of Time Stamps in Handling Latency and Concept Drift in Online Learning" by Marrs, Black and Hickey describes an online classification framework that can operate under concept drift and that can be subject to latency in example arrival. Two algorithms are derived from such a framework. They are further evaluated on data streams, compared and their performance is discussed.

Paper 4 "Sliding Mode Incremental Learning Algorithm for Interval Type-2 Takagi-Sugeno-Kang Fuzzy Neural Networks" by Ahmed et al. proposes an online learning algorithm for interval type-2 Takagi-Sugeno-Kang (TSK) fuzzy neural networks in a way that type-2 fuzzy rules are incrementally extracted using a sliding mode. Moreover, the algorithm is tested and compared against the stateof-the-art sliding mode on-line learning algorithms for both type-1 and type-2 fuzzy neural networks.

Paper 5 "Negotiating in Dynamic Environments: TimeEfficient Automated Negotiations by Means of Combinatorial Auctions" by Lang and Fink investigates the problem of automated negotiation in the context of heterogeneous intelligent agents. For dynamic adaptation, smart negotiation protocols that ensure good social welfare and strategyproof are required. Three protocols are proposed and are, respectively, based on: combinatorial auctions, self-selective clustering of agents in combinatorial auctions and iterative negotiation. The experiments show that the first protocol is well suited as a method for negotiation in dynamic environments.

Paper 6 "Middleware for Task Resolution and Adaptation in Pervasive Environments" by Ben Lahmar, Belaid and Mukhtar discusses the problem of adaptation in pervasive environments. Basically it investigates the issue of matching and selecting software and hardware components to execute tasks in a pervasive context. In particular, the authors propose a middleware/algorithm that finds for a given task the best device and component used for its execution. Relying on a monitoring mechanism, the changes are captured and new devices and components are dynamically and accordingly selected.

The last paper 7 "A Fuzzy Logic Model based Markov Random Field for Medical Image Segmentation" by Nguyen and $\mathrm{Wu}$ proposes an adaptive fuzzy inference system for image segmentation. Markov random field (MRF) is applied to take the neighborhood between pixels used along with clique potential Markov random field (MRF) in the premises of the fuzzy if-then rules. The role of MRF is to capture local spatial information of pixels during the rule induction process. The hybrid method is evaluated on simulated and real brain images.

At the end, the Guest editor wishes to express his gratitude to the editor-in-chief and the editorial office assistants for their valuable support throughout the process of review and publication of the present special issue. 